\title{
Curcumin attenuates hypoxic-ischemic brain injury in neonatal rats through induction of nuclear factor erythroid-2-related factor 2 and heme oxygenase-1
}

\author{
XIAOLU CUI ${ }^{1}$, HONGQUAN SONG ${ }^{2}$ and $\mathrm{JIE} \mathrm{SU}^{3}$ \\ ${ }^{1}$ Department of Rehabilitation Medicine, Shandong Provincial Qianfoshan Hospital, Jinan, \\ Shandong 250014; ${ }^{2}$ Department of Spleen and Stomach, Affiliated Hospital of Shandong University of TCM, \\ Jinan, Shandong 250013; ${ }^{3}$ Department of Cadres and Health Care, The Second Affiliated Hospital of \\ Shandong University of TCM, Jinan, Shandong 250001, P.R. China
}

Received October 29, 2015; Accepted March 3, 2017

DOI: $10.3892 /$ etm.2017.4683

\begin{abstract}
Curcumin has previously demonstrated anti-inflammatory, anti-infective and immuno-suppressive effects. In the present study, whether the attenuating effects of curcumin against hypoxic-ischemic brain injury in neonatal rats are mediated via nuclear factor erythroid-2-related factor 2 (Nrf2) and heme oxygenase-1 (HO-1) was investigated. A model of hypoxic-ischemic brain injury was created using 1-week-old Sprague Dawley rats (weight, $52 \pm 1 \mathrm{~g}$ ). The model rats were treated with $150 \mathrm{mg} / \mathrm{kg}$ curcumin by gavage for 3 days. Malondialdehyde levels, and superoxide dismutase and caspase- 3 activities were assayed using commercial kits and western blot analysis was used to measure inducible nitric oxide synthase (iNOS), Nrf2 and HO-1 expression levels. Treatment with curcumin effectively reduced the brain injury score, increased myelin basic protein (MBP) expression and increased the quantity of neuronal cells in neonatal rats with hypoxic-ischemic brain injury. Furthermore, treatment with curcumin significantly attenuated the changes in SOD activity and MDA levels and suppressed the iNOS protein expression induced in neonatal rats by hypoxic-ischemic brain injury. Treatment with curcumin significantly increased Nrf2 and HO-1 expression in the neonatal rats with hypoxic-ischemic brain injury. The present study indicated that curcumin attenuates hypoxic-ischemic brain injury in neonatal rats through the induction of Nrf2 and HO-1.
\end{abstract}

Correspondence to: Mr. Jie Su, Department of Cadres and Health Care, The Second Affiliated Hospital of Shandong University of TCM, 1 West Qingnian Road, Central Road, Jinan, Shandong 250001, P.R. China

E-mail: jiesuss123@163.com

Key words: curcumin, hypoxic-ischemic brain injury, nuclear factor erythroid-2-related factor 2, heme oxygenase-1

\section{Introduction}

Hypoxic ischemic encephalopathy (HIE) is fetal or neonatal cerebral injury caused by partial or complete anoxia and a reduction or suspension of the cerebral blood flow resulting from perinatal asphyxia, which has chronic sequelae on the nerve system and is a cause of acute neonatal death (1). Early detection, diagnosis and intervention of HIE is important for the reduction of perinatal mortality and disability rates for newborns (2).

Nuclear factor erythroid-2-related factor $2(\mathrm{Nrf} 2)$ is a nuclear transcription factor with neuroprotective effects against central nervous system disease (3). The Nrf2 pathway is anti-apoptotic and has multiple functions, including resistance to oxidative stress, regulation of inflammatory injury and relief from calcium overloading (4). Research findings indicate that Nrf2 regulates downstream molecules to protect nerves, particularly against apoptosis (5).

Heme oxygenase-1 (HO-1) is an induced enzyme with a relative molecular mass of $32 \mathrm{kDa}$, which is a major heat shock/stress protein, mainly distributed among tissues and organs with active metabolism of blood corpuscles, such as the spleen, liver, reticuloendothelial system and bone marrow (6). HO-1 has low expression levels in brain tissues; however, under stress conditions, HO-1 can be expressed at very high levels by neurogliocytes and stellate neurons (7). Stimulating factors such as heat shock, heavy metals, protoheme, inflammatory stimuli, hypoxia, ultraviolet radiation, endotoxins, prostaglandin, lipopolysaccharides, nitric oxide (NO) and stress states such as hunger, fever and mechanical injuries may increase HO-1 activity (8).

Curcumin has been applied in traditional medicine for thousands of years, particularly in Ayurvedic medicine (9). It has been demonstrated to possess a wide range of pharmacological activities with little side effects and high safety. Extensive studies have been conducted on curcumin, which have demonstrated that curcumin has significant anti-infection, anti-inflammatory, antioxidative, anti-platelet aggregation, lipid-reducing and anti-fibrotic activities, in addition to protecting against myocardial injury and suppressing 
tumor growth (9-11). Curcumin has been shown to ameliorate myocardial ischemia-reperfusion injuries and protect the myocardium through inhibition of the activation of inflammation-related genes by NF- $\mathrm{B}$ (12). Thus, in the present study, the effects of curcumin on hypoxic-ischemic brain injury and the potential underlying mechanisms were investigated.

\section{Materials and methods}

Animals and hypoxia-ischemia induction. All animal experiments were approved by the Animal Ethics Committee of the Second Affiliated Hospital of Shandong University of TCM (Jinan, China). Male Sprague Dawley rats (1-week-old; weight, $52 \pm 1 \mathrm{~g} ; \mathrm{n}=50$ ) were purchased from the Animal Laboratory of Shandong University (Jinan, China), and housed with a 12:12-h light-dark cycle, a temperature of $22-23^{\circ} \mathrm{C}$ and $55-60 \%$ humidity, with free access to a standard laboratory chow diet and drinking water.

The hypoxia-ischemia model was created as follows. Briefly, rats were anesthetized with $2 \%$ isoflurane (Sigma-Aldrich; Merck KGaA, Darmstadt, Germany) in a 1:1 mixture of nitrous oxide and oxygen. The left common carotid arteries of rats were ligated and the rats was allowed to recover for $1 \mathrm{~h}$. After recovery, rats were placed in an incubator perfused with $10 \pm 0.01 \%$ oxygen in nitrogen at $37^{\circ} \mathrm{C}$ for $1 \mathrm{~h}$. In the hemisphere ipsilateral to the left common carotid artery, arterial ligation and hypoxia resulted in the creation of the HIE model. In the sham group, rats were only anesthetized and did not undergo surgery to induce HIE.

Experimental design. Rats were randomly divided into three groups: Sham group $(n=10)$, hypoxic-ischemic brain injury model group $(n=20)$ and curcumin group $(n=20)$. In the curcumin group, rats subjected to hypoxic-ischemic brain injury were treated with $150 \mathrm{mg} / \mathrm{kg}$ /day curcumin (Sigma-Aldrich; Merck KGaA, Darmstadt, Germany) by gavage for 3 days, 2 days after HIE surgery. In the sham group and the hypoxic-ischemic brain injury model group, rats were treated with $500 \mu 1$ normal saline at the same time points. Rats were sacrificed by cervical dislocation while anesthetized with isoflurane after treatment with curcumin.

Assessment of brain damage. After sacrifice, the brains were dissected, paraffin-embedded and cut into $10-\mu \mathrm{m}$ coronal sections. Myelin basic protein (MBP) was stained, and the extent of white- and gray-matter injury was analyzed via quantitative measurements of the injury area using Micro Image version 4.0 (Micro-Macro AB, Gothenburg, Sweden), with the following formula: Contralateral hemisphere-ipsilateral hemisphere $)=($ contralateral hemisphere $) \times 100 \%$. The sections were dipped in $1 \%$ thionin/toluidine solution, soaked in acid fuchsin solution and dehydrated in a gradient of ethanol.

Histopathology. Following sacrifice, the brain was immediately removed and fixed with $10 \%$ neutral buffered formalin at room temperature for $24 \mathrm{~h}$. Tissue samples were cut into sections of 4- $\mu \mathrm{m}$ thickness and stained with hematoxylin and eosin (H\&E). Tissue samples were observed using confocal microscope (magnification, x20; ZEISS, LSM510; Zeiss AG, Oberkochen, Germany).
Analysis of superoxide dismutase (SOD) activities and malondialdehyde (MDA) levels. SOD activities and MDA levels were assayed using ELISA kits (A001-2 and A003-1; Nanjing Jiancheng Biology Engineering Institute, Nanjing, China) and absorbance's were measured using an ultraviolet spectrophotometer at $450 \mathrm{~nm}$.

Western blot analysis. Brain samples were transferred into ice-cold RIPA assay (Beyotime Institute of Biotechnology; Shanghai, China) containing a cocktail of protein phosphatase and proteinase inhibitors. Samples were collected at 15,000 x g at $4^{\circ} \mathrm{C}$ for $10 \mathrm{~min}$. The supernatant was assayed using a Bio-Rad Bradford Protein Assay kit (Bio-Rad Laboratories, Inc., Hercules, CA, USA). Protein $(50 \mu \mathrm{g})$ was separated using 8-10\% SDS-PAGE and blots were blocked in buffer solution containing $5 \%$ milk and $0.1 \%$ Tween-20 in PBS, following transfer onto polyvinylidene fluoride membranes. Membranes was then blotted for $2 \mathrm{~h}$ at room temperature with antibodies targeting inducible nitric oxide synthase (iNOS; 1:4,000; Sigma-Aldrich; Merck KGaA), Nrf2 (1:4,000; Sigma-Aldrich; Merck KGaA), HO-1 (1:4,000; Sigma-Aldrich; Merck KGaA) or $\beta$-actin (1:4,000; Sigma-Aldrich; Merck KGaA) at $4^{\circ} \mathrm{C}$ overnight. Membranes were washed with TBST for $15 \mathrm{~min}$ and incubated with goat anti-rabbit HRP secondary antibody (1:3,000; HA-1001-100; Hangzhou Hua'An Biotechnology Co., Ltd., Hangzhou, China) for $1 \mathrm{~h}$ at $37^{\circ} \mathrm{C}$. Protein expression was detected using an enhanced chemiluminescence kit (Beyotime Institute of Biotechnology, Shanghai, China) and quantified using Bio-Rad Image Lab 3.0 (Bio-Rad).

Caspase-3 activity assay. Brain samples were transferred into ice-cold Tris-HCl buffer solution containing a cocktail of protein phosphatase and proteinase inhibitors. Samples were collected at $15,000 \times \mathrm{g}$ at $4^{\circ} \mathrm{C}$ for $10 \mathrm{~min}$. The supernatant was assayed using a Bio-Rad Bradford Protein Assay kit. An equal amount of total protein $(10 \mu \mathrm{g})$ extract from each rat was incubated at $37^{\circ} \mathrm{C}$ with Ac-LEHD-pNA for the caspase-3 assay (Beyotime Institute of Biotechnology; Shanghai, China) for $4 \mathrm{~h}$ and the caspase- 3 activity was determined by measuring the absorbance at $405 \mathrm{~nm}$ using an ultraviolet spectrophotometer.

Statistical analysis. All results are expressed as the mean \pm standard deviation. Analysis of variance followed by the least significant difference post hoc test was used for the comparison of data from more than two groups using SPSS software (17.0; SPSS, Inc., Chicago, IL, USA). P<0.05 was considered to indicate a statistically significant difference.

\section{Results}

Curcumin attenuates hypoxic-ischemic brain injury in neonatal rats. The chemical structure of curcumin is displayed in Fig. 1. The effects of curcumin on hypoxic-ischemic brain injury were examined by calculation of a brain injury score. The brain injury score of the model group was significantly increased compared with that of the sham control group (Fig. 2). In the rats treated with curcumin $(150 \mathrm{mg} / \mathrm{kg})$, the brain injury induced by hypoxic-ischemic brain injury was reduced compared with that in the model group (Fig. 2). 
Curcumin attenuates MBP levels in brain-injured neonatal rats. To evaluate whether curcumin attenuates changes in MBP expression in neonatal rats, MBP levels were analyzed following hypoxic-ischemic brain injury. As shown in Fig. 3, the level of MBP in the model group was lower than that of the sham control group. Treatment with $150 \mathrm{mg} / \mathrm{kg}$ curcumin effectively increased the MBP levels of neonatal rats with hypoxic-ischemic brain injury (Fig. 3).

Curcumin attenuates histopathological changes in brain-injured neonatal rats. To confirm the effects of curcumin in neonatal rats, histopathological examination was conducted using H\&E staining. The number of neuronal cells in the hypoxic-ischemic brain injury model group was lower than that of the sham group (Fig. 4). Treatment with curcumin effectively augmented the quantity of neuronal cells in neonatal rats with hypoxic-ischemic brain injury (Fig. 4).

Curcumin attenuates changes in SOD activity and MDA levels in brain-injured neonatal rats. To determine whether curcumin attenuates oxidative stress in neonatal rats, SOD activities and MDA levels were measured using ELISA kits. As shown in Fig. 5A, the SOD activity of the hypoxic-ischemic brain injury model group was clearly inhibited, as compared with that of the sham control group. In addition, hypoxic-ischemic brain injury observably increased MDA levels in neonatal rats, as compared with those in the sham control group (Fig. 5B). However, treatment with curcumin observably reversed the changes in SOD activity and MDA levels in neonatal rats following hypoxic-ischemic brain injury (Fig. 5).

Curcumin attenuates the expression of iNOS in brain-injured neonatal rats. To evaluate the attenuating effects of curcumin on the expression of iNOS in neonatal rats, iNOS expression was analyzed using western blot analysis. Following hypoxic-ischemic brain injury, iNOS protein expression was markedly increased in the model group, as compared with the sham control group (Fig. 6). Treatment with curcumin significantly suppressed iNOS protein expression in neonatal rats with hypoxic-ischemic brain injury (Fig. 6).

Curcumin increases the expression of Nrf2 in brain-injured neonatal rats. To further evaluate the effects of curcumin in neonatal rats, the expression of Nrf2 protein was analyzed using western blot analysis. The Nrf2 protein expression level in the sham group was similar to that of the model control group (Fig. 7). However, curcumin treatment markedly promoted Nrf2 protein expression in neonatal rats with hypoxic-ischemic brain injury (Fig. 7).

Curcumin attenuates the expression of $\mathrm{HO}-1$ in brain-injured neonatal rats. The effects of curcumin on neonatal rats with hypoxic-ischemic brain injury were further analyzed using western blotting analysis to determine HO-1 protein levels. As shown in Fig. 8, the protein expression of HO-1 in the sham group was similar to that in the model control group. Following curcumin treatment, there was a significant upregulation of HO-1 protein expression in the curcumin treated group, as compared with the model group (Fig. 8).<smiles>COc1cc(/C=C/C(=O)CC(=O)/C=C/c2ccc(O)c(OC)c2)ccc1O</smiles>

Figure 1. Chemical structure of curcumin

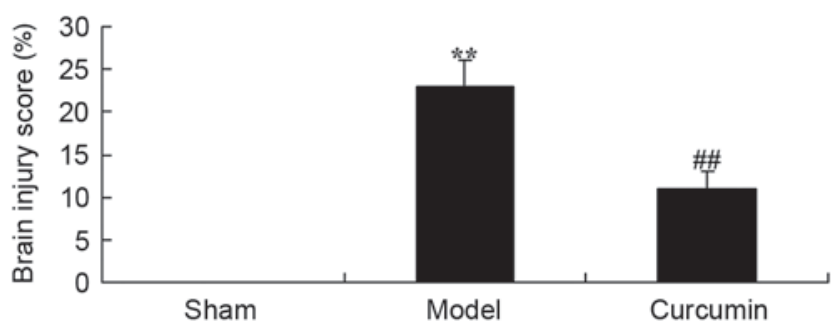

Figure 2. Curcumin attenuates hypoxic-ischemic brain injury in neonatal rats, as demonstrated by a reduction in the brain injury score. ${ }^{* *} \mathrm{P}<0.01$ vs. the sham group; ${ }^{\# \#} \mathrm{P}<0.01$ vs. the model group. Sham, sham group; model, hypoxic-ischemic brain injury model group; curcumin, curcumin treated group.

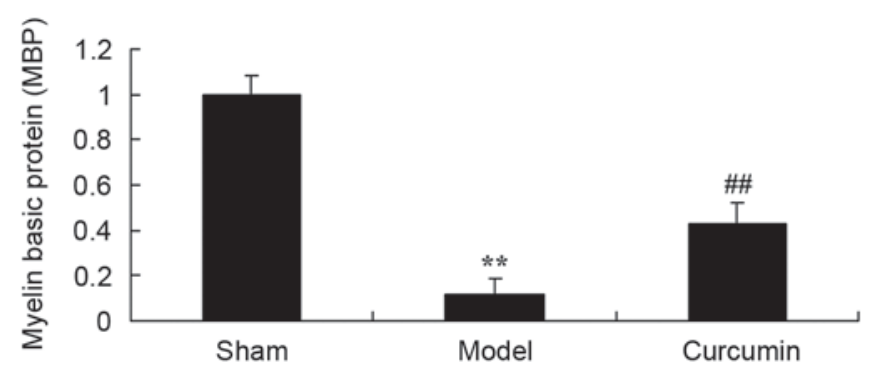

Figure 3. Curcumin attenuates the reduction in MBP levels induced by hypoxic-ischemic brain injury in neonatal rats. ${ }^{* *} \mathrm{P}<0.01$ vs. the sham group; ${ }^{\# \#} \mathrm{P}<0.01$ vs. the hypoxic-ischemic brain injury model group. MBP, myelin basic protein; sham, sham group; model, hypoxic-ischemic brain injury model group; curcumin, curcumin treated group.

Curcumin attenuates the activity of caspase-3 in brain-injured neonatal rats. To further investigate the effects of curcumin in neonatal rats, the activity of caspase-3 was determined. The caspase- 3 activity was significantly increased in the hypoxic-ischemic brain injury model group, as compared with the sham control group (Fig. 9). Curcumin treatment significantly inhibited the caspase- 3 activity in neonatal rats with hypoxic-ischemic brain injury (Fig. 9).

\section{Discussion}

The occurrence rate of HIE for term infants has been reported to be $6 / 1,000$, and 1/1,000 of such infants succumb or have nervous system dysfunction (13). Each year, China has 18-20 million live births and the morbidity of asphyxia has reached as high as $13.6 \%$, among which $15.6 \%$ of cases are injured or disabled (14). Each year, 300,000 children are handicapped, which greatly threatens their quality of life (15). In order to better prevent injury and disability, the timely detection and treatment of HIE is necessary, and the exploration 


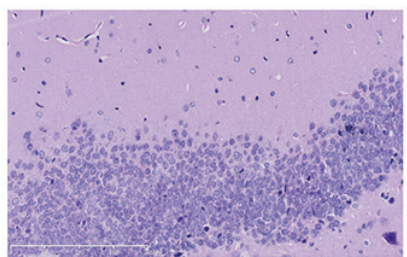

Sham

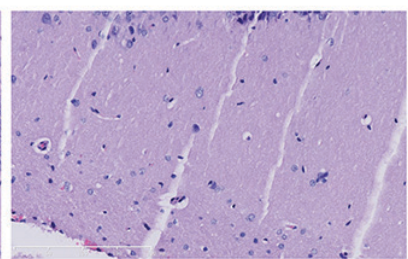

Model

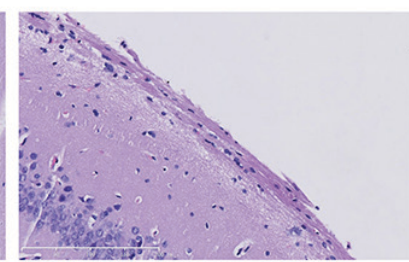

Curcumin

Figure 4. Curcumin attenuates the histopathological changes induced by hypoxic-ischemic brain injury in neonatal rats. Magnification, $\mathrm{x} 20$. Scale bar=200 $\mu \mathrm{m}$. Sham, sham group; model, hypoxic-ischemic brain injury model group; curcumin, curcumin treated group.

A

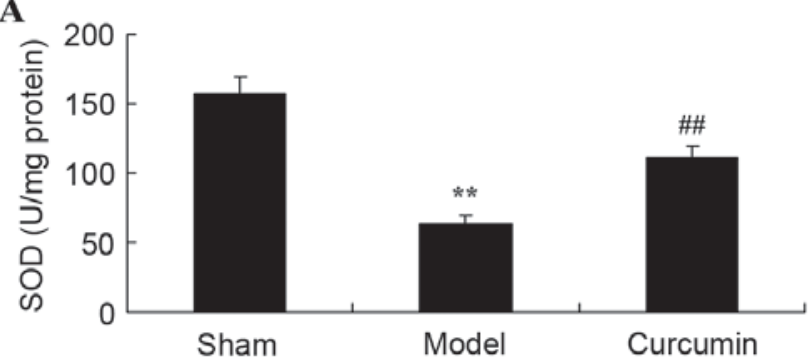

B

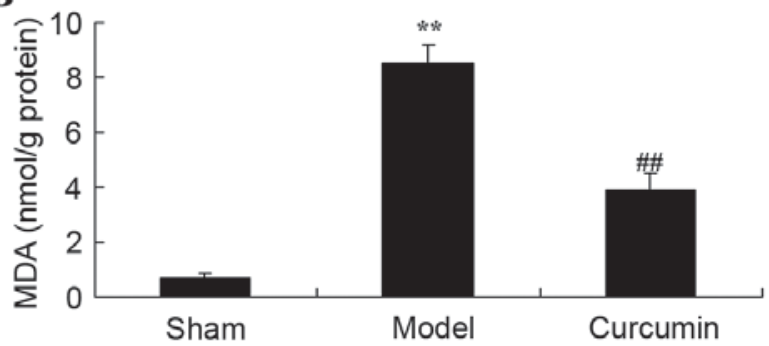

Figure 5. Curcumin attenuates the changes in SOD activity and MDA levels induced by hypoxic-ischemic brain injury in neonatal rats. Effects of curcumin on (A) SOD activity and (B) MDA levels in neonatal rats. ${ }^{* *} \mathrm{P}<0.01$ vs. the sham group; ${ }^{\# \#} \mathrm{P}<0.01$ vs. the hypoxic-ischemic brain injury model group. SOD, superoxide dismutase; MDA, malondialdehyde; sham, sham group; model, hypoxic-ischemic brain injury model group; curcumin, curcumin treated group.

A

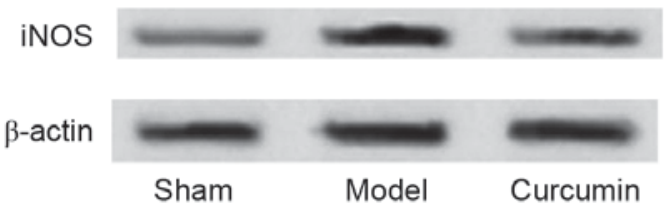

B

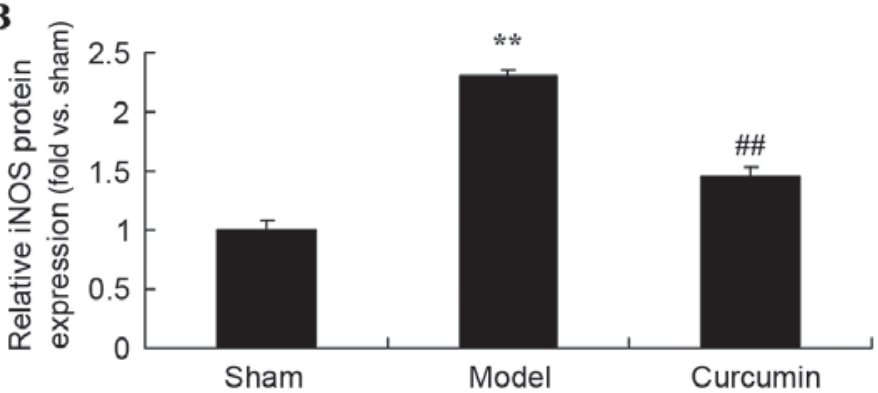

Figure 6. Curcumin attenuates the expression of iNOS induced by hypoxic-ischemic brain injury in neonatal rats. Effect of curcumin on the expression of iNOS protein as shown (A) using western blot analysis with (B) quantitative analysis of iNOS protein expression in neonatal rats. ${ }^{* *} \mathrm{P}<0.01$ vs. the sham group; ${ }^{\# \#} \mathrm{P}<0.01$ vs. the hypoxic-ischemic brain injury model group. iNOS, inducible nitric oxide synthase; sham, sham group; model, hypoxic-ischemic brain injury model group; curcumin, curcumin treated group.

A

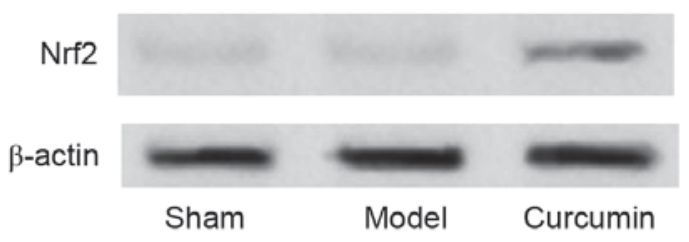

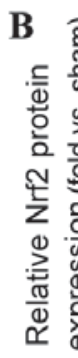

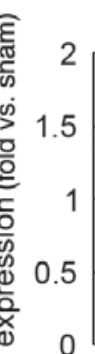

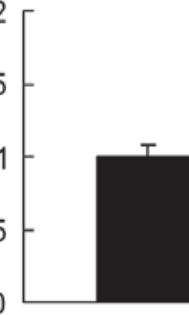

Sham

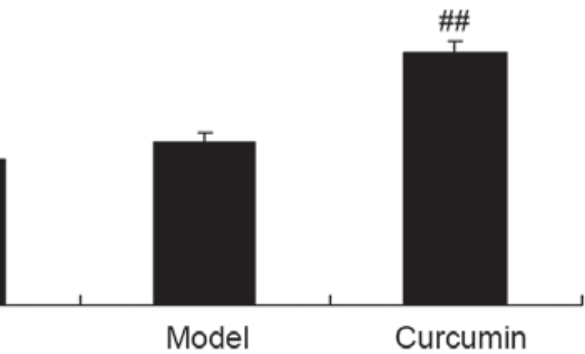

Figure 7. Curcumin increases the expression of Nrf2 in neonatal rats with hypoxic-ischemic brain injury. Effect of curcumin on the expression of Nrf2 protein as shown (A) using western blot analysis with (B) quantitative analysis of Nrf2 protein expression in neonatal rats. ${ }^{\# \#} \mathrm{P}<0.01$ vs. the hypoxic-ischemic brain injury model group. Nrf2, nuclear factor erythroid-2-related factor 2; sham, sham group; model, hypoxic-ischemic brain injury model group; curcumin, curcumin treated group.

of indicators or methods with relatively high specificity is required (16). In the present study, treatment with curcumin was evaluated and was found to effectively reduce brain injury score, increase MBP levels and augment the quantity 
A

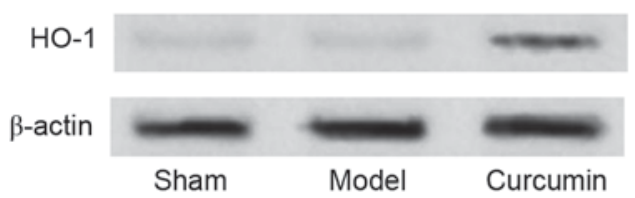

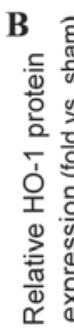

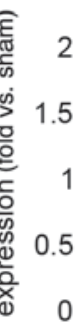

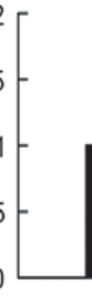

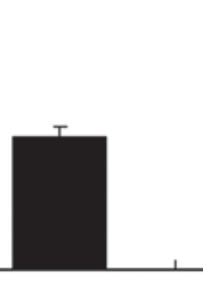

Sham

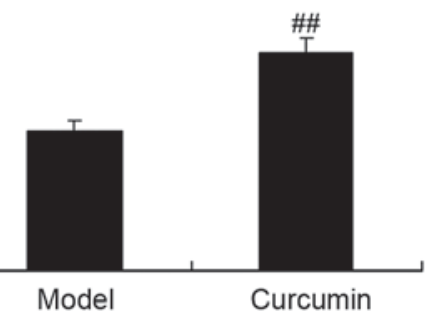

Figure 8. Curcumin increases the expression of HO-1 in neonatal rats with hypoxic-ischemic brain injury. Effect of curcumin on the expression of HO-1 protein expression as shown (A) using western blot analysis and (B) quantitative analysis of $\mathrm{HO}-1$ protein expression in neonatal rats. ${ }^{\# \#} \mathrm{P}<0.01 \mathrm{vs}$. the hypoxic-ischemic brain injury model group. HO-1, heme oxygenase-1; sham, sham group; model, hypoxic-ischemic brain injury model group; curcumin, curcumin treated group.

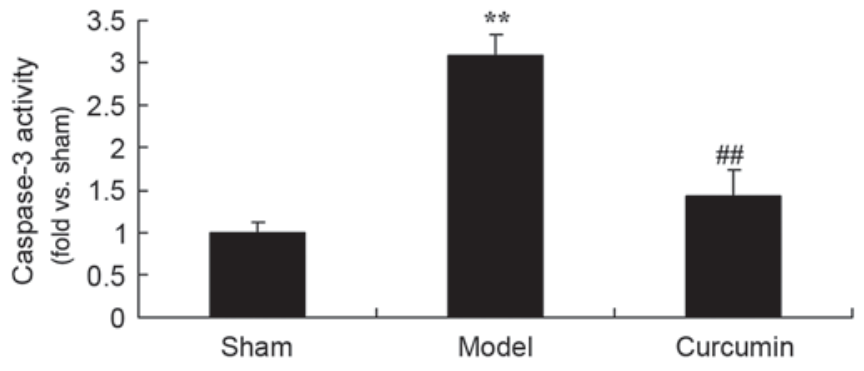

Figure 9. Curcumin attenuates the elevated activity of caspase-3 induced by hypoxic-ischemic brain injury in neonatal rats. ${ }^{* *} \mathrm{P}<0.01$ vs. the sham group; ${ }^{\#} \mathrm{P}<0.01$ vs. the hypoxic-ischemic brain injury model group. Sham, sham group; model, hypoxic-ischemic brain injury model group; curcumin, curcumin treated group.

of neuronal cells in neonatal rats with hypoxic-ischemic brain injury.

When HIE occurs, oxidative phosphorylation dysfunction of mitochondria results in a reduction of ATP production and produces a large amount of reactive oxygen species (ROS), resulting in oxidative stress (17). With regard to cerebral ischemia reperfusion, certain adaptive regulatory mechanisms in the HIE process have attracted attention (18). Following HIE, the oxidation and anti-oxidation system of the body is unbalanced. High levels of free radicals are generated, which results in damage, i.e., oxidative stress (19). Free radicals are ROS, including superoxide anion and hydrogen peroxide, which can cause the peroxidation of lipid, proteins and nucleic acids (20). Neurons have high metabolic activities and oxygen consumption as well as low levels of endogenous anti-oxidant enzymes, and are extremely sensitive to oxidative stress (21). In addition, abundant lipids exist in the brain and can react with ROS, resulting in lipid peroxidation of neural membranes (22). Consequently, the central nervous system is sensitive to oxidative stress injuries. In the present study, it was found that treatment with curcumin observably reversed the changes in SOD activities and MDA levels in neonatal rats with hypoxic-ischemic brain injury. Similar mechanisms have been indicated in the cardiovascular system; Qian et al (9) suggested that curcumin mitigates cardiac injury through suppression of oxidation and inflammation in obese rats.

To exert their physiological functions, vascular endothelial cells secrete various vasoactive substances. Free radicals are damaging to endothelial cells. Autologous vascular wall cells undergo stress reactions and exert resistance to oxidative stress. NO is an endothelium-derived relaxing factor (23). When oxidative stress occurs, the expression of NO synthase decreases, with concurrent reduction of its substrate and cofactors (24). A reduction in the activity of NO synthase and the degradation of NO by active oxygen can both cause abnormalities in NO levels (22). The present study demonstrated that treatment with curcumin clearly suppressed the elevation of iNOS expression induced by hypoxic-ischemic brain injury in neonatal rats. Gao et al (25) reported that curcumin induces M2 macrophage polarization by iNOS.

Recent studies have shown that neuronal apoptosis has an essential role in secondary injury following traumatic brain injury $(26,27)$. The Nrf2 pathway is an anti-apoptotic pathway that has been demonstrate do function in many organs, particularly in the central nervous system (5). With a short half-life, Nrf2 is an important factor. Under physiological conditions, Nrf2 exists in the cytoplasm where it binds with Keap1 protein (28). When oxidative stress from external stimuli occurs, phosphorylation of Nrf2 separates it from the adapter protein Keap1 and transfers it into the cell nucleus to combine with the promoter sequence to regulate its downstream genes (29). The Nrf2 anti-apoptotic pathway has multiple functions relating to protection against anti-oxidative stress, regulation of inflammatory injury and relief from calcium overloading (30). In the present study, curcumin treatment markedly promoted Nrf2 protein expression in neonatal rats with hypoxic-ischemic brain injury. Zhang et al (31) suggested that curcumin protects renal tubular epithelial cells through the Nrf2-mediated upregulation of HO-1.

HO-1 is a member of the heat shock protein family, which is able to stabilize tissue proteins, mitochondrial membranes and cytomembranes and relieve neurocyte injuries (7). Following stress, heat shock proteins are typically located perinuclearly and may combine with plasma membranes to stabilize them (27). The stability of brain cell functions and structures is dependent upon plasma membranes (32). It has been found that increased $\mathrm{HO}-1$ expression provides resistance against neuronal death mediated via glutamic acid-induced oxidative stress, and significantly reduces membrane damage and nuclear accumulation (28). Furthermore, when HO-1 levels increase, the calcium ion concentration in resting cells decreases and the production rate of oxygen radicals is largely reduced (4). In the present study, it was found that curcumin treatment significantly upregulated HO-1 protein expression in neonatal rats with hypoxic-ischemic brain injury. When considered together, these findings suggest that the 
Nrf2-mediated upregulation of HO-1 may play a detrimental role in hypoxic-ischemic brain injury.

Apoptosis is a programmed death under physiological or pathological conditions. Three pathways of cell apoptosis have been described, i.e., the death receptor pathway in cytomembranes, the mitochondrial pathway in cytomembranes and stress pathways of the endoplasmic reticulum $(13,33)$. The latter two pathways have been investigated via the measurement ofcaspase- 3 and caspase-12 activities in a study on cell apoptosis in a neonatal hypoxia-ischemia rat model (34). In the present study, curcumin treatment significantly inhibited caspase-3 activity in the brains of neonatal rats with hypoxic-ischemic brain injury. In a previous study, Bucak et al (35) demonstrated that the protective effect of curcumin against paclitaxel-induced inner ear damage in rats was also mediated via the suppression of caspase-3 activity.

In summary, the present study provides evidence in a 1-week-old Sprague Dawley rat model confirming the protective effects of curcumin against hypoxic-ischemic brain injury in neonatal rats. The results of the study indicate that the Nrf2/HO-1 pathway underlies the protective effect of curcumin on hypoxic-ischemic brain injury, and reveal the effective targeting of $\mathrm{Nrf} 2 / \mathrm{HO}-1$ by curcumin.

\section{References}

1. Shankaran S, Laptook AR, Pappas A, McDonald SA, Das A Tyson JE, Poindexter BB, Schibler K, Bell EF, Heyne RJ, et al: Effect of depth and duration of cooling on deaths in the NICU among neonates with hypoxic ischemic encephalopathy: A randomized clinical trial. JAMA 312: 2629-2639, 2014.

2. Kracer B, Hintz SR, Van Meurs KP and Lee HC: Hypothermia therapy for neonatal hypoxic ischemic encephalopathy in the state of California. J Pediatr 165: 267-273, 2014.

3. Re L, Martinez-Sãnchez G, Bordicchia M, Malcangi G, Pocognoli A, Morales-Segura MA, Rothchild J and Rojas A: Is ozone pre-conditioning effect linked to Nrf2/EpRE activation pathway in vivo? A preliminary result. Eur J Pharmacol 742: 158-162, 2014.

4. Wu Z, Uchi H, Morino-Koga S, Shi W and Furue M: Z-ligustilide ameliorated ultraviolet B-induced oxidative stress and inflammatory cytokine production in human keratinocytes through upregulation of $\mathrm{Nrf} 2 / \mathrm{HO}-1$ and suppression of NF- $\mathrm{KB}$ pathway. Exp Dermatol 24: 703-708, 2015.

5. Sasaki H, Suzuki A, Shitara M, Hikosaka Y, Okuda K, Moriyama S, Yano M and Fujii Y: Polymorphisms of NRF2 gene correlated with decreased FEV1 in lung cancers of smokers. Biomed Rep 1: 484-488, 2013.

6. Barnett M, Hall S, Dixit M and Arany I: Simvastatin attenuates oleic acid-induced oxidative stress through CREB-dependent induction of heme oxygenase-1 in renal proximal tubule cells. Pediatr Res 79: 243-250, 2016.

7. Lin SH, Song W, Cressatti M,Zukor H, Wang E and Schipper HM: Heme oxygenase-1 modulates microRNA expression in cultured astroglia: Implications for chronic brain disorders. Glia 63: 1270-1284, 2015.

8. Morita K, Itoh M, Nishibori N, Her S and Lee MS: Spirulina non-protein components induce BDNF gene transcription via HO-1 activity in C6 glioma cells. Appl Biochem Biotechnol 175: 892-901, 2015

9. Qian Y, Zhong P, Liang D, Xu Z, Skibba M, Zeng C, Li X, Wei T, Wu L and Liang G: A newly designed curcumin analog Y20 mitigates cardiac injury via anti-inflammatory and anti-oxidant actions in obese rats. PLoS One 10: e0120215, 2015.

10. Patel PB, Thakkar VR and Patel JS: Cellular effect of curcumin and citral combination on breast cancer cells: Induction of apoptosis and cell cycle arrest. J Breast Cancer 18: 225-234, 2015.

11. Jang EM, Choi MS, Jung UJ, Kim MJ, Kim HJ, Jeon SM, Shin SK, Seong CN and Lee MK: Beneficial effects of curcumin on hyperlipidemia and insulin resistance in high-fat-fed hamsters. Metabolism 57: 1576-1583, 2008.
12. Yeh $\mathrm{CH}$, Chen TP, $\mathrm{Wu} \mathrm{YC}$, Lin $\mathrm{YM}$ and Jing Lin P: Inhibition of NFkappaB activation with curcumin attenuates plasma inflammatory cytokines surge and cardiomyocytic apoptosis following cardiac ischemia/reperfusion. J Surg Res 125: 109-116, 2005.

13. Liu L, Liu C, Lu Y, Liu L and Jiang Y: ER stress related factor ATF6 and caspase-12 trigger apoptosis in neonatal hypoxic-ischemic encephalopathy. Int J Clin Exp Pathol 8: 6960-6966, 2015.

14. Kaandorp JJ, Benders MJ, Rademaker CM, Torrance HL, Oudijk MA, de Haan TR, Bloemenkamp KW, Rijken M, van Pampus MG, Bos AF, et al: Antenatal allopurinol for reduction of birth asphyxia induced brain damage (ALLO-Trial); a randomized double blind placebo controlled multicenter study. BMC Pregnancy Childbirth 10: 8, 2010.

15. Bell MJ, Adelson PD, Hutchison JS, Kochanek PM, Tasker RC, Vavilala MS, Beers SR, Fabio A, Kelsey SF and Wisniewski SR; Multiple Medical Therapies for Pediatric Traumatic Brain Injury Workgroup: Differences in medical therapy goals for children with severe traumatic brain injury-an international study. Pediatr Crit Care Med 14: 811-818, 2013.

16. Agut T, Leon M, Rebollo M, Muchart J, Arca G and Garcia-Alix A: Early identification of brain injury in infants with hypoxic ischemic encephalopathy at high risk for severe impairments: Accuracy of MRI performed in the first days of life. BMC Pediatr 14: 177, 2014.

17. Zhao M, Zhu P, Fujino M, Zhuang J, Guo H, Sheikh I, Zhao L and Li XK: Oxidative stress in hypoxic-ischemic encephalopathy: Molecular mechanisms and therapeutic strategies. Int J Mol Sci 17: pii: E2078, 2016.

18. Vasiljević B, Maglajlić-Djukić S, Gojnić M and Stanković S: The role of oxidative stress in perinatal hypoxic-ischemic brain injury. Srp Arh Celok Lek 140: 35-41, 2012.

19. Burchell SR, Dixon BJ, Tang J and Zhang JH: Isoflurane provides neuroprotection in neonatal hypoxic ischemic brain injury. J Investig Med 61: 1078-1083, 2013.

20. Zhu C, Xu F, Fukuda A, Wang X, Fukuda H, Korhonen L, Hagberg H, Lannering B, Nilsson M, Eriksson PS, et al: X chromosome-linked inhibitor of apoptosis protein reduces oxidative stress after cerebral irradiation or hypoxia-ischemia through up-regulation of mitochondrial antioxidants. Eur J Neurosci 26: 3402-3410, 2007

21. Perrone S, Tataranno LM, Stazzoni G, Ramenghi L and Buonocore G: Brain susceptibility to oxidative stress in the perinatal period. J Matern Fetal Neonatal Med 28: (Suppl 1): S2291-S2295, 2015.

22. Chang CC, Wang YH, Chern CM, Liou KT, Hou YC, Peng YT and Shen YC: Prodigiosin inhibits gp91(phox) and iNOS expression to protect mice against the oxidative/nitrosative brain injury induced by hypoxia-ischemia. Toxicol Appl Pharmacol 257: 137-147, 2011.

23. Sosunov SA, Ameer X, Niatsetskaya ZV, Utkina-Sosunova I, Ratner VI and Ten VS: Isoflurane anesthesia initiated at the onset of reperfusion attenuates oxidative and hypoxic-ischemic brain injury. PLoS One 10: e0120456, 2015.

24. Feng Y, Lu S, Wang J, Kumar P, Zhang L and Bhatt AJ: Dexamethasone-induced neuroprotection in hypoxic-ischemic brain injury in newborn rats is partly mediated via Akt activation. Brain Res 1589: 68-77, 2014.

25. Gao S, Zhou J, Liu N, Wang L, Gao Q, Wu Y, Zhao Q, Liu P, Wang S, Liu Y, et al: Curcumin induces M2 macrophage polarization by secretion IL-4 and/or IL-13. J Mol Cell Cardiol 85: 131-139, 2015.

26. Zhao H, Mitchell S, Ciechanowicz S, Savage S, Wang T, Ji X and Ma D: Argon protects against hypoxic-ischemic brain injury in neonatal rats through activation of nuclear factor (erythroid-derived 2)-like 2. Oncotarget 7: 25640-25651, 2016.

27. Zhai X, Lin H, Chen Y, Chen X, Shi J, Chen O, Li J and Sun X: Hyperbaric oxygen preconditioning ameliorates hypoxia-ischemia brain damage by activating Nrf 2 expression in vivo and in vitro. Free Radic Res 50: 454-466, 2016.

28. Li B, Choi HJ, Lee DS, Oh H, Kim YC, Moon JY, Park WH, Park SD and Kim JE: Amomum tsao-ko suppresses lipopolysaccharide-induced inflammatory responses in RAW264.7 macrophages via Nrf2-dependent heme oxygenase-1 expression. Am J Chin Med 42: 1229-1244, 2014.

29. Yang H, Xu W, Zhou Z, Liu J, Li X, Chen L, Weng J and Yu Z: Curcumin attenuates urinary excretion of albumin in type II diabetic patients with enhancing nuclear factor erythroid-derived 2-like 2 (Nrf2) system and repressing inflammatory signaling efficacies. Exp Clin Endocrinol Diabetes 123: 360-367, 2015. 
30. Rzepecka J, Pineda MA, Al-Riyami L, Rodgers DT, Huggan JK Lumb FE, Khalaf AI, Meakin PJ, Corbet M, Ashford ML, et al: Prophylactic and therapeutic treatment with a synthetic analogue of a parasitic worm product prevents experimental arthritis and inhibits IL-1 $\beta$ production via NRF2-mediated counter-regulation of the inflammasome. J Autoimmun 60: 59-73, 2015.

31. Zhang X, Liang D, Guo L, Liang W, Jiang Y, Li H, Zhao Y, Lu S and Chi ZH: Curcumin protects renal tubular epithelial cells from high glucose-induced epithelial-to-mesenchymal transition through Nrf2-mediated upregulation of heme oxygenase-1. Mol Med Rep 12: 1347-1355, 2015.

32. Zimmermann K, Baldinger J, Mayerhofer B, Atanasov AG, Dirsch VM and Heiss EH: Activated AMPK boosts the Nrf2/HO-1 signaling axis-A role for the unfolded protein response. Free Radic Biol Med 88: 417-426, 2015.
33. Askalan R, Gabarin N, Armstrong EA, Fang Liu Y, Couchman D and Yager JY: Mechanisms of neurodegeneration after severe hypoxic-ischemic injury in the neonatal rat brain. Brain Res 1629: 94-103, 2015.

34. Cai J, Kang Z, Liu WW, Luo X, Qiang S, Zhang JH, Ohta S, Sun X, Xu W, Tao H and Li R: Hydrogen therapy reduces apoptosis in neonatal hypoxia-ischemia rat model. Neurosci Lett 441: 167-172, 2008.

35. Bucak A, Ozdemir C, Ulu S, Gonul Y, Aycicek A, Uysal M and Cangal A: Investigation of protective role of curcumin against paclitaxel-induced inner ear damage in rats. Laryngoscope 125: 1175-1182, 2015. 